Bull. Soc. math. France

133 (2), 2005, p. 275-295

\title{
SUR LE RANG DES JACOBIENNES SUR UN CORPS DE FONCTIONS
}

\author{
PAR Marc Hindry \& Amílcar PaCheco
}

\begin{abstract}
RÉSUMÉ. - Soit $f: \mathcal{X} \rightarrow C$ une surface projective fibrée au-dessus d'une courbe et définie sur un corps de nombres $k$. Nous donnons une interprétation du rang du groupe de Mordell-Weil sur $k(C)$ de la jacobienne de la fibre générique (modulo la partie constante) en termes de moyenne des traces de Frobenius sur les fibres de $f$. L'énoncé fournit une réinterprétation de la conjecture de Tate pour la surface $\mathcal{X}$ et généralise des résultats de Nagao, Rosen-Silverman et Wazir.

AbStract ( On the rank of Jacobians on a function field). - Let $f: \mathcal{X} \rightarrow C$ be a projective surface fibered over a curve and defined over a number field $k$. We give an interpretation of the rank of the Mordell-Weil group over $k(C)$ of the jacobian of the generic fibre (modulo the constant part) in terms of average of the traces of Frobenius on the fibers of $f$. The results also give a reinterpretation of the Tate conjecture for the surface $\mathcal{X}$ and generalizes results of Nagao, Rosen-Silverman and Wazir.
\end{abstract}

Texte reçu le 21 avril 2003, révisé le 8 octobre 2003, accepté le 7 novembre 2003

Marc Hindry, Université Denis Diderot Paris VII, U.F.R. Mathématiques, case 7012, 2 Place Jussieu, 75251 Paris (France) • E-mail : hindry@math.jussieu.fr

Amílcar Pacheco, Universidade Federal do Rio de Janeiro (Universidade do Brasil), Departamento de Matemática Pura, Rua Guaiaquil 83, Cachambi, 20785-050 Rio de Janeiro RJ(Brasil) • E-mail : amilcar@impa.br

Classification mathématique par sujets (2000). — 11G, 11G40, 11M, $14 \mathrm{G} 10$.

Mots clefs. - Variété jacobienne, corps de fonctions, conjecture de Tate.

Amílcar Pacheco a été partiellement soutenu par la bourse de recherche CNPq 300896/91-3 et par le projet PRONEX 41.96.0830.00. Ce travail a commencé pendant une visite du premier auteur au deuxième auteur à l'IMPA dans le cadre de l'accord Brésil-France 69.0014/01-5.

BULletiN DE LA SOCIÉTÉ MATHÉMATIQUE DE FRANCE

(C) Société Mathématique de France

$0037-9484 / 2005 / 275 / \$ 5.00$ 


\section{Introduction}

Soient $k$ un corps de nombres, $\mathcal{X}$ une surface projective lisse irréductible sur $k, C$ une courbe projective lisse irréductible sur $k, f: \mathcal{X} \rightarrow C$ un morphisme propre plat tel que les fibres soient des courbes de genre arithmétique $g \geq 1$; ces hypothèses entraînent que la fibre générique est lisse et irréductible. Soient $K=k(C)$ le corps de fonctions de $C, X / K$ la fibre générique de $f, J_{X}$ la variété jacobienne de $X$ et $(\tau, B)$ la $K / k$-trace de $J_{X}$. Un théorème de Lang-Néron affirme que $J_{X}(k(C)) / \tau(B(k))$ et même $J_{X}(\bar{k}(C)) / \tau(B(\bar{k}))$ sont des groupes de type fini. Shioda [15] a donné une interprétation du deuxième groupe comme quotient du groupe de Néron-Severi de la surface $\mathcal{X}$ par un sousgroupe explicite. Nous allons discuter une interprétation du rang du premier groupe en termes de l'arithmétique de la surface $\mathcal{X}$.

Soit $S$ un ensemble fini d'idéaux premiers de l'anneau d'entiers $\mathcal{O}_{k}$ de $k$ tel que pour tout $\mathfrak{p} \notin S, \mathcal{X}$ et $C$ aient bonne réduction modulo $\mathfrak{p}$ et que la réduction $f_{\mathfrak{p}}$ de $f$ modulo $\mathfrak{p}$ soit un morphisme propre et plat $f_{\mathfrak{p}}: \mathcal{X}_{\mathfrak{p}} \rightarrow C_{\mathfrak{p}}$ ayant pour fibres des courbes de genre arithmétique $g$ sur le corps résiduel $\kappa_{\mathfrak{p}}$ de $\mathfrak{p}$ avec $q_{\mathfrak{p}}$ éléments. Pour chaque $c \in C_{\mathfrak{p}}$, soit $\mathcal{X}_{\mathfrak{p}, c}=f_{\mathfrak{p}}^{-1}(c)$ la fibre de $f_{\mathfrak{p}}$ en $c$.

Notation. - Soient $\ell$ un corps, $\bar{\ell}$ une clôture algébrique de $\ell$ et $V$ une variété algébrique projective lisse définie sur $\ell$. Notons $\bar{V}=V \times_{\ell} \bar{\ell}$. Si $\ell$ est parfait, notons aussi $G_{\ell}=\operatorname{Gal}(\bar{\ell} / \ell)$.

Soient $F_{\mathfrak{p}} \in G_{k}$ un élément de Frobenius et $I_{\mathfrak{p}} \subset G_{k}$ son groupe d'inertie. Soit $\Delta=\left\{c \in C \mid \mathcal{X}_{\mathfrak{p}, c}\right.$ est singulière $\}$ le lieu discriminant de $f$. Après avoir élargi $S$ si nécessaire, on peut supposer que pour tout $\mathfrak{p} \notin S$ le lieu discriminant $\Delta_{\mathfrak{p}}$ de $f_{\mathfrak{p}}$ est égal à la réduction du lieu discriminant de $f$ modulo $\mathfrak{p}$.

Soit $\bar{F}_{\mathfrak{p}}$ l'automorphisme de Frobenius sur $H_{\text {ét }}^{1}\left(\overline{\mathcal{X}}_{\mathfrak{p}, c}, \mathbb{Q}_{\ell}\right)$ induit par l'automorphisme de Frobenius géométrique de $\kappa_{\mathfrak{p}}$. Si $c \in\left(C_{\mathfrak{p}}-\Delta_{\mathfrak{p}}\right)\left(\kappa_{\mathfrak{p}}\right)$, on définit

$$
a_{\mathfrak{p}}\left(\mathcal{X}_{\mathfrak{p}, c}\right)=\operatorname{Tr}\left(\bar{F}_{\mathfrak{p}} \mid H_{\text {ét }}^{1}\left(\overline{\mathcal{X}}_{\mathfrak{p}, c}, \mathbb{Q}_{\ell}\right)\right) \text {. }
$$

Si $c \in \Delta_{\mathfrak{p}}\left(\kappa_{\mathfrak{p}}\right)$, on remplace $H_{\text {ét }}^{1}$ par le groupe $H_{\underline{c}}^{1}$ de cohomologie $\ell$-adique à support propre, i.e., $a_{\mathfrak{p}}\left(\mathcal{X}_{\mathfrak{p}, c}\right)=\operatorname{Tr}\left(\bar{F}_{\mathfrak{p}} \mid H_{\underline{c}}^{1}\left(\overline{\mathcal{X}}_{\mathfrak{p}, c}, \overline{\mathbb{Q}}_{\ell}\right)\right)$.

Soit $a_{\mathfrak{p}}(B)=\operatorname{Tr}\left(F_{\mathfrak{p}} \mid H_{\text {ét }}^{1}\left(\bar{B}, \mathbb{Q}_{\ell}\right)^{I_{\mathfrak{p}}}\right)$. Après avoir ajouté un nombre fini d'idéaux premiers de $k$ à $S$ on peut supposer que, pour tout $\mathfrak{p} \notin S$, la variété $B$ ait bonne réduction $B_{\mathfrak{p}}$ modulo $\mathfrak{p}$. Dans ce cas là, $a_{\mathfrak{p}}(B)=\operatorname{Tr}\left(\bar{F}_{\mathfrak{p}} \mid H_{\text {ét }}^{1}\left(\bar{B}_{\mathfrak{p}}, \mathbb{Q}_{\ell}\right)\right)$. On définit alors la trace moyenne de Frobenius

$$
A_{\mathfrak{p}}(\mathcal{X})=\frac{1}{q_{\mathfrak{p}}} \sum_{c \in C_{\mathfrak{p}}\left(\kappa_{\mathfrak{p}}\right)} a_{\mathfrak{p}}\left(\mathcal{X}_{\mathfrak{p}, c}\right)
$$

ainsi que trace moyenne réduite

$$
A_{\mathfrak{p}}^{*}(\mathcal{X})=A_{\mathfrak{p}}(\mathcal{X})-a_{\mathfrak{p}}(B) .
$$

TOME $133-2005-\mathrm{N}^{\mathrm{O}} 2$ 
Soit $L_{2}(\mathcal{X} / k, s)=\prod_{\mathfrak{p}} \operatorname{det}\left(1-F_{\mathfrak{p}} q_{\mathfrak{p}}^{-s} \mid H_{\text {ét }}^{2}\left(\overline{\mathcal{X}}, \mathbb{Q}_{\ell}\right)^{I_{\mathfrak{p}}}\right)^{-1}$ la fonction $L$ de $\mathcal{X}$ associée à $H_{\text {ét }}^{2}\left(\overline{\mathcal{X}}, \mathbb{Q}_{\ell}\right)$. Soient $\operatorname{Pic}(\mathcal{X})$ le groupe des classes de diviseurs de $\mathcal{X}, \operatorname{Pic}^{0}(\mathcal{X})$ le sous-groupe de diviseurs algébriquement équivalents à zéro, $\operatorname{NS}(\mathcal{X})=\operatorname{Pic}(\mathcal{X}) / \operatorname{Pic}^{0}(\mathcal{X})$ le groupe de Néron-Severi de $\mathcal{X}$ et $\mathrm{NS}(\mathcal{X} / k)$ le sous-groupe de classes de diviseurs de $\operatorname{NS}(\mathcal{X})$ qui sont définies sur $k$. Notons que si $z$ est fixé par $G_{k}$, i.e., $z \in \mathrm{NS}(\mathcal{X})^{G_{k}}$, il existe un multiple $n z$ de $z$ tel que $n z \in \mathrm{NS}(\mathcal{X} / k)$. Donc, $\mathrm{NS}(\mathcal{X})^{G_{k}} \otimes \mathbb{Q} \cong \mathrm{NS}(\mathcal{X} / k) \otimes \mathbb{Q}$ et en particulier $\operatorname{rang}\left(\mathrm{NS}(\mathcal{X})^{G_{k}}\right)=\operatorname{rang}(\mathrm{NS}(\mathcal{X} / k))$.

Conjecture 1.1 (conjecture de Tate [16]). — La fonction $L_{2}(\mathcal{X} / k, s)$ possède un pôle en $s=2$ d'ordre $\operatorname{rang}(\mathrm{NS}(\mathcal{X} / k))$.

Remarque 1.2. - Il s'agit d'une version de la conjecture de Tate pour les diviseurs, la conjecture générale concerne tous les cycles algébriques.

1) On peut se dispenser de l'hypothèse d'un prolongement méromorphe au voisinage de $s=2$ en interprétant la phrase « $L_{2}(\mathcal{X} / k, s)$ possède un pôle d'ordre $t$ en $s=2 »$ comme signifiant

$$
\lim _{\substack{s \rightarrow 2 \\ \operatorname{Re}(s)>2}}(s-2)^{t} L_{2}(\mathcal{X} / k, s)=\alpha \neq 0 .
$$

De même, si $f(s)$ est holomorphe sur $\operatorname{Re}(s)>\lambda$ et si

$$
\lim _{s \rightarrow \lambda}(s-\lambda) f(s)=\alpha \neq 0,
$$

on appellera $\alpha$ le résidu de la fonction $f(s)$ en $s=\lambda$ et on écrira Rés $s_{s=\lambda} f(s)=$ $\alpha$.

2) Dans la plupart des cas où l'on sait démontrer un prolongement analytique de $L_{2}(\mathcal{X} / k, s)$ à la droite $\operatorname{Re}(s)=2$, on sait également démontrer que la fonction ne s'annule pas sur cette droite. Cette propriété est importante car elle permet d'appliquer un théorème taubérien $[7, \mathrm{ch}$. XV] à la dérivée logarithmique de $L_{2}(\mathcal{X} / k, s)$.

Le but de ce travail est de prouver le théorème suivant.

ThÉORÈme 1.3. - La conjecture 1.1 pour la surface $\mathcal{X}$ implique

$$
\operatorname{Rés}_{s=1}\left(\sum_{\mathfrak{p} \notin S}-A_{\mathfrak{p}}^{*}(\mathcal{X}) \frac{\log \left(q_{\mathfrak{p}}\right)}{q_{\mathfrak{p}}^{s}}\right)=\operatorname{rang}\left(\frac{J_{X}(K)}{\tau B(k)}\right) .
$$

Sous l'hypothèse additionelle que $L_{2}(\mathcal{X} / k, s)$ se prolonge analytiquement sur la droite $\operatorname{Re}(s)=2$ et n'a pas de zéros sur cette droite, on conclut aussi que

$$
\lim _{T \rightarrow \infty} \frac{1}{T}\left(\sum_{\substack{\mathfrak{p} \notin S \\ q_{\mathfrak{s}} \leq T}}-A_{\mathfrak{p}}^{*}(\mathcal{X}) \log \left(q_{\mathfrak{p}}\right)\right)=\operatorname{rang}\left(\frac{J_{X}(K)}{\tau B(k)}\right) .
$$


Remarque 1.4. - En fait l'égalité (1.1) est essentiellement équivalente à la conjecture de Tate 1.1, voir le corollaire 5.8. L'égalité (1.1) est une généralisation de la conjecture analytique de Nagao [14, Nagao's Conjecture 1.1'] et (1.2) de la conjecture taubérienne de Nagao [14, Nagao's Conjecture 1.1] telles qu'elles sont formulées par Rosen et Silverman pour les fibrations de genre 1 admettant une section; lorsque de plus $C=\mathbb{P}^{1}$ et $k=\mathbb{Q}$, la forme taubérienne est la conjecture originelle, due à Nagao [10], [11] (nous préférons appeler «taubérienne » la forme de la conjecture que Rosen et Silverman appellent «arithmetic »).

Remarque 1.5. - Le premier travail dans la direction du théorème 1.3 est dû à Nagao [11] qui a formulé la conjecture taubérienne à partir de calculs explicites dans le cas où $g=1$ et $f$ admet une section (donc $X$ est une courbe elliptique) et $K=\mathbb{Q}(x)$. Son objectif, atteint dans plusieurs cas, était de produire des courbes elliptiques sur $\mathbb{Q}$ avec rang « assez grand». Par la suite, Rosen et Silverman dans [14, th. 1.3] on traité le cas général où $K=k(C)$ est le corps de fonctions d'une courbe lisse et projective sur $k$, toujours avec $X$ courbe elliptique. Ils ont formulé la conjecture analytique et l'ont reliée avec la formulation taubérienne, montrant le théorème 1.3 dans ce cas. Plus tard, Wazir [19, th. 1.1] a généralisé le résultat de Rosen-Silverman au cas d'une variété $\mathcal{X}$ de dimension 3 fibrée au-dessus d'une surface en courbes elliptiques. On peut s'attendre à ce que l'énoncé du théorème reste vrai pour toute fibration en courbe $f: \mathcal{X} \rightarrow T$ de genre au moins 1 avec $n=\operatorname{dim} \mathcal{X}=\operatorname{dim} T+1$ mais nous nous contenterons ici de traiter le cas des surfaces.

\section{Conjecture de Birch et Swinnerton Dyer et conjecture de Tate}

Ce paragraphe contient les motivations pour le théorème principal ainsi que des considérations de nature spéculative; on explique en particulier pourquoi il est nécessaire de remplacer la trace moyenne $A_{\mathfrak{p}}(\mathcal{X})$ par la trace moyenne « réduite » $A_{\mathfrak{p}}^{*}(\mathcal{X})$ pour espérer un énoncé du type (1.2).

Notation. - Comme il est d'usage, pour deux fonctions $f(t)$ et $g(t)$ définies au voisinage de $\omega$, on écrira, $f(t)=O(g(t))$ si il existe un voisinage de $\omega$ et une constante $C$ tels que $|f(t)| \leq C g(t)$ sur ce voisinage. On écrira $f(t)=o(g(t))$ si $\lim _{t \rightarrow \omega} f(t) / g(t)=0$.

Si $\mathcal{X}$ est une variété lisse projective, définie sur un corps de nombres $k$, ayant bonne réduction hors d'un ensemble fini de places $S$, on notera, en négligeant un nombre fini de facteurs eulériens

$$
L_{2}(\mathcal{X} / k, s)=\prod_{\mathfrak{p} \notin S} \operatorname{det}\left(1-F_{\mathfrak{p}} q_{\mathfrak{p}}^{-s} \mid H_{\text {êt }}^{2}\left(\mathcal{X} \times_{k} \bar{k}, \mathbb{Q}_{\ell}\right)\right)^{-1} .
$$

TOME $133-2005-\mathrm{N}^{\mathrm{O}} 2$ 
Pour $\mathfrak{p}$ ne divisant pas le conducteur, les valeurs propres du Frobenius $F_{\mathfrak{p}}$ sont de modules $q_{\mathfrak{p}}$. Le produit d'Euler converge pour $\operatorname{Re}(s)>2$. La conjecture 1.1 donne une interprétation du rang du groupe de Néron-Severi $\mathrm{NS}(\mathcal{X})$ en termes de cette fonction. La conjecture 1.1 est en fait formulée pour une variété de dimension quelconque $[13,5.5]$.

Si $A$ est une variété abélienne définie sur un corps de nombres $k$, on notera simplement sa fonction $L$ :

$$
L(A / k, s)=\prod_{\mathfrak{p}} \operatorname{det}\left(1-F_{\mathfrak{p}} q_{\mathfrak{p}}^{-s} \mid H_{\text {ét }}^{1}\left(A \times_{k} \bar{k}, \mathbb{Q}_{\ell}\right)^{I_{\mathfrak{p}}}\right)^{-1} .
$$

Pour $\mathfrak{p}$ ne divisant pas le conducteur, les valeurs propres du Frobenius $F_{\mathfrak{p}}$, disons $\alpha_{\mathfrak{p}, j}$, sont de modules $q_{\mathfrak{p}}^{1 / 2}$. Le produit d'Euler converge pour $\operatorname{Re}(s)>\frac{3}{2}$. La conjecture centrale de la théorie donne une interprétation du rang du groupe de Mordell-Weil $A(k)$ en termes de cette fonction.

Conjecture 2.1 (conjecture de Birch et Swinnerton-Dyer [16])

Soit $A$ une variété abélienne définie sur un corps de nombres $k$. La fonction $L(A / k, s)$ admet un prolongement analytique à $\mathbb{C}$ et possède en $s=1$ un zéro d'ordre $\operatorname{rang}(A(k))$.

Remarque 2.2. - Il s'agit ici de la première partie de la conjecture; nous ne discuterons pas la seconde partie qui décrit le coefficient dominant de $L(A / k, s)$ en $s=1$.

Un calcul simple et classique montre que

$$
\begin{aligned}
-\frac{\mathrm{d}}{\mathrm{d} s}(\log (L(A / k, s))) & =\sum_{\mathfrak{p}, m \geq 1} \sum_{j} \alpha_{\mathfrak{p}, j}^{m} \log \left(q_{\mathfrak{p}}\right) q_{\mathfrak{p}}^{-m s} \\
& =\sum_{\mathfrak{p}} a_{\mathfrak{p}}(A) \log \left(q_{\mathfrak{p}}\right) q_{\mathfrak{p}}^{-s}+h_{1}(s) \\
& =\sum_{\mathfrak{p}} a_{\mathfrak{p}}(A) \log \left(q_{\mathfrak{p}}\right) q_{\mathfrak{p}}^{-s}+\sum_{\mathfrak{p}} \sum_{j} \alpha_{\mathfrak{p}, j}^{2} \log \left(q_{\mathfrak{p}}\right) q_{\mathfrak{p}}^{-2 s}+h_{2}(s)
\end{aligned}
$$

avec $h_{1}(s)$ holomorphe sur $\operatorname{Re}(s)>1$ et $h_{2}(s)$ holomorphe sur $\operatorname{Re}(s)>\frac{5}{6}$. Définissons

$$
M(A / k, s)=\sum_{\mathfrak{p}}-a_{\mathfrak{p}}(A) \log \left(q_{\mathfrak{p}}\right) q_{\mathfrak{p}}^{-s}
$$

ainsi que la fonction arithmétique correspondante

$$
M_{A}(T)=\sum_{q_{\mathfrak{p}} \leq T}-a_{\mathfrak{p}}(A) \log \left(q_{\mathfrak{p}}\right) .
$$

Alors le prolongement analytique de $L(A / k, s)$ jusqu'à $\operatorname{Re}(s)=1$ équivaut à celui de $M(A / k, s)$ et un zéro d'ordre $r$ en $s=1$ pour $L(A / k, s)$ équivaut à un pôle simple pour $M(A / k, s)$ en $s=1$ avec résidu $r$. Remarquons aussi 
que l'holomorphie de $M(A / k, s)$ pour $\operatorname{Re}(s)>1$ équivaut à l'holomorphie de $L^{\prime}(A / k, s) / L(A / k, s)$ pour $\operatorname{Re}(s)>1$ et donc à l'hypothèse de Riemann généralisée pour $L(A / k, s)$ ou encore, par des arguments analytiques classiques à la propriété $M_{A}(T)=O_{\epsilon}\left(T^{1+\epsilon}\right)$ pour tout $\epsilon>0$. De plus il semble raisonnable de conjecturer que $h_{1}(s)$ devrait être holomorphe sur $\operatorname{Re}(s)=1$; cela proviendrait de l'holomorphie et la non annulation $\operatorname{sur} \operatorname{Re}(s)=2$ de la fonction $\prod_{\mathfrak{p}, j}\left(1-\alpha_{\mathfrak{p}, j}^{2} q_{\mathfrak{p}}^{-s}\right)^{-1}$, ce qui est démontré dans le cas où $A$ est modulaire (par la méthode de Rankin, complétée par Shimura).

Acceptant cela, on peut alors s'interroger sur la vraisemblance de l'équivalence $M_{A}(T) \sim r T$; en effet, d'une part, si cette équivalence était vraie alors on en déduirait facilement que $M(A / k, s)$ aurait un pôle simple en $s=1$ avec résidu $r$ et donc la conjecture de Birch et Swinnerton-Dyer serait vérifiée pour $A / k$, si $r=\operatorname{rang}(A(k))$, d'autre part, même en supposant l'hypothèse de Riemann généralisée et la conjecture de Birch et Swinnerton-Dyer pour $L(A / k, s)$, on ne peut en déduire par les arguments taubériens classiques l'équivalence $M_{A}(T) \sim r T$ à cause de la présence d'une infinité de pôles pour $M(A / k, s)$ (resp. de zéros pour $L(A / k, s)$ ) sur la droite $\operatorname{Re}(s)=1$. La présence d'une infinité de pôles rend même fort peu vraisemblable l'équivalence $M_{A}(T) \sim r T$. On conclut ces considérations en notant qu'il semble donc illusoire d'espérer remplacer la conclusion du théorème principal par

$$
\lim _{T \rightarrow \infty} \frac{1}{T} \sum_{q_{\mathfrak{p}} \leq T}-A_{\mathfrak{p}}(\mathcal{X}) \log q_{\mathfrak{p}}=\operatorname{rang}\left(J_{X}(K)\right)
$$

(sauf, bien sûr, dans le cas où $B$ est nulle). Par contre la version analytique

$$
\operatorname{Rés}_{s=1}\left(\sum_{\mathfrak{p} \notin S}-A_{\mathfrak{p}}(\mathcal{X}) \frac{\log \left(q_{\mathfrak{p}}\right)}{q_{\mathfrak{p}}^{s}}\right)=\operatorname{rang}\left(J_{X}(K)\right)
$$

découlerait des conjectures de Tate pour $\mathcal{X}$ et Birch et Swinnerton-Dyer pour $B$. En effet, si l'on admet le prolongement analytique, alors le résidu en $s=1$ est égal à

$$
\begin{aligned}
\operatorname{rang}\left(J_{X}(K)\right)+(\underset{s=1}{\operatorname{ord}}(L(B / k, s))- & \operatorname{rang}(B(k))) \\
& -\left(\operatorname{rang}(\operatorname{NS}(\mathcal{X} / k))+\underset{s=2}{\operatorname{ord}}\left(L_{2}(\mathcal{X} / k, s)\right)\right) .
\end{aligned}
$$

Ainsi la situation est plus favorable sur les corps de fonctions; cela est dû au fait que, du point de vue de la théorie analytique des nombres, on effectue une double moyenne et que l'on doit considérer le comportement d'une série de Dirichlet au bord de son demi-plan de convergence, et non à l'intérieur de la bande critique.

Remarque 2.3. - Il serait peut-être plus naturel, au lieu de définir la moyenne des traces de Frobenius $A_{\mathfrak{p}}(\mathcal{X})$ comme nous l'avons fait (imitant en 
cela Nagao [11] et Rosen-Silverman [14]), de poser

$$
A_{\mathfrak{p}}^{\prime}(\mathcal{X})=\frac{1}{\# C_{\mathfrak{p}}\left(\kappa_{\mathfrak{p}}\right)} \sum_{c \in C_{\mathfrak{p}}\left(\kappa_{\mathfrak{p}}\right)} a_{\mathfrak{p}}\left(\mathcal{X}_{c}\right) \quad \text { et } \quad A_{\mathfrak{p}}^{*}(\mathcal{X}):=A_{\mathfrak{p}}^{\prime}(\mathcal{X})-a_{\mathfrak{p}}(B)
$$

On peut observer qu'une telle modification ne changerait pas les énoncés en vue. En effet, on voit aisément que l'égalité voulue

$$
\lim _{T \rightarrow \infty} \frac{1}{T} \sum_{\substack{\mathfrak{p} \notin S \\ q_{\mathfrak{p}} \leq T}}\left(A_{\mathfrak{p}}^{*}(\mathcal{X})-A_{\mathfrak{p}}^{\prime *}(\mathcal{X})\right) \log \left(q_{\mathfrak{p}}\right)=0
$$

est vérifiée si et seulement si

$$
N(T):=\sum_{\substack{\mathfrak{p} \notin S \\ q_{\mathfrak{p}} \leq T}} a_{\mathfrak{p}}(C) A_{\mathfrak{p}}^{*}(\mathcal{X}) \frac{\log \left(q_{\mathfrak{p}}\right)}{q_{\mathfrak{p}}}=o(T) .
$$

Cette dernière estimation est donc vraie si $C=\mathbb{P}^{1}$. Observons maintenant que $A_{\mathfrak{p}}^{*}(\mathcal{X})$, qui est trivialement $O\left(q_{\mathfrak{p}}^{1 / 2}\right)$ (comme moyenne des $a_{\mathfrak{p}}\left(\mathcal{X}_{c}\right)$ qui sont $O\left(q_{\mathfrak{p}}^{1 / 2}\right)$ ) est en fait un $O(1)$ à cause de la formule (4.7) (démontrée plus loin au paragraphe 4 , où chaque terme est défini) qui indique que

$$
A_{\mathfrak{p}}^{*}(\mathcal{X})=-\frac{b_{\mathfrak{p}}(\mathcal{X})}{q_{\mathfrak{p}}}+\operatorname{Tr}\left(F_{\mathfrak{p}} \mid \mathcal{S}\right)+O\left(q_{\mathfrak{p}}^{-1 / 2}\right) .
$$

Or $b_{p}(\mathcal{X})$ est la trace d'un opérateur avec valeurs propres de module $q_{\mathfrak{p}}$ donc $b_{\mathfrak{p}}(\mathcal{X})=O\left(q_{\mathfrak{p}}\right)$, alors que $F_{\mathfrak{p}}$ agit sur $\mathcal{S}$ avec pour valeurs propres des racines de l'unité (i.e., de façon quasi-unipotente), donc on a bien $A_{\mathfrak{p}}^{*}(\mathcal{X})=O(1)$. Ainsi on obtient

$N(T)=\sum_{q_{\mathfrak{p}} \leq T} a_{\mathfrak{p}}(C) A_{\mathfrak{p}}^{*}(\mathcal{X}) \frac{\log q_{\mathfrak{p}}}{q_{\mathfrak{p}}} \ll \sum_{q_{\mathfrak{p}} \leq T}\left|a_{\mathfrak{p}}(C)\right| \frac{\log q_{\mathfrak{p}}}{q_{\mathfrak{p}}} \ll \sum_{q_{\mathfrak{p}} \leq T} \frac{\log q_{\mathfrak{p}}}{\sqrt{q_{\mathfrak{p}}}} \ll \log T \sqrt{T}$

\section{Outils géométriques}

Tout comme dans Rosen-Silverman [14], l'idée de la preuve est de compter le nombre des points rationnels de $\mathcal{X}_{\mathfrak{p}}$ sur $\kappa_{\mathfrak{p}}$ de deux manières. La première consiste à employer la formule de Lefschetz pour $\mathcal{X}_{\mathfrak{p}}$, la deuxième à compter le nombre des points dans chaque fibre $\mathcal{X}_{\mathfrak{p}, c}$ pour $c \in C_{\mathfrak{p}}\left(\kappa_{\mathfrak{p}}\right)$ et calculer la somme. Pour obtenir ces deux résultats on a besoin d'une part d'un lemme géométrique (lemme 3.2) qui permet de déterminer la cardinalité de $\mathcal{X}_{\mathfrak{p}, c}\left(\kappa_{\mathfrak{p}}\right)$ et d'autre part d'une formule (proposition 3.8) analogue à celle de Shioda-Tate pour les surfaces elliptiques, généralisée par Shioda aux fibrations de genre supérieur admettant une section [15, th. 1]. On développe ces résultats dans ce paragraphe. Pour obtenir le premier, nous généralisons les calculs explicites au cas par cas de [14], [19] par le Lemme 3.2. Enfin nous incluons une preuve de la formule de Shioda-Tate [15] car, d'une part, nous ne souhaitons pas faire l'hypothèse que la 
fibration possède une section, d'autre part, le fait de travailler en caractéristique zéro simplifie la preuve (les schémas en groupes, notamment $\mathrm{Pic}^{0}$, sont réduits).

Nous commençons par rappeler le lemme suivant qui indique quelques propriétés des fibres singulières de familles de courbes ( $c f .[1])$.

LEMME 3.1 (voir $[1,(1.1)]$ ). — Soit $R$ un anneau de valuation discrète avec corps résiduel algébriquement clos $\kappa$. Soit $\mathcal{V}$ un schéma régulier de dimension 2 propre sur $\operatorname{Spec}(R)$. On suppose que la fibre fermée $V$ soit connexe et de dimension 1. En tant que sous-schéma de $\mathcal{V}, V$ est un diviseur de Cartier effectif que l'on écrit sous la forme $V=\sum_{i=1}^{n} r_{i} C_{i}$. La matrice d'intersection $\left\|\left(C_{i} \cdot C_{j}\right)\right\|$ définit une forme quadratique dont le noyau est engendré par $V$ et qui est définie négative sur $\left(\bigoplus_{i=1}^{n} \mathbb{Q} C_{i}\right) / \mathbb{Q} V$.

Un point clef dans notre argument est le lemme suivant. Nous remercions le referee pour nous avoir indiqué la preuve de ce lemme (nous proposions une preuve beaucoup plus compliquée, basé sur l'analyse des fibres spéciales singulières donnée par Artin et Winters [1], qui de plus ne donnait le résultat qu'à $O\left(q_{\mathfrak{p}}^{1 / 2}\right)$ près $)$.

LEMME 3.2. - Soient $\mathfrak{p} \notin S, c \in C_{\mathfrak{p}}\left(\kappa_{\mathfrak{p}}\right), \mathcal{X}_{\mathfrak{p}, c}=f_{\mathfrak{p}}^{-1}(c)$ et $m_{c}$ le nombre des composantes $\kappa_{\mathfrak{p}}$-rationnelles de $\mathcal{X}_{\mathfrak{p}, c}$. Le nombre de points $\kappa_{\mathfrak{p}}$-rationnels de $\mathcal{X}_{\mathfrak{p}, c}$ s'exprime comme suivant :

$$
\# \mathcal{X}_{\mathfrak{p}, c}\left(\kappa_{\mathfrak{p}}\right)=1-a_{\mathfrak{p}}\left(\mathcal{X}_{\mathfrak{p}, c}\right)+q_{\mathfrak{p}} m_{c}
$$

Démonstration. - Par le théorème de Weil, pour tout $c \in\left(C_{\mathfrak{p}}-\Delta_{\mathfrak{p}}\right)\left(\kappa_{\mathfrak{p}}\right)$ on a

$$
\# \mathcal{X}_{\mathfrak{p}, c}\left(\kappa_{\mathfrak{p}}\right)=q_{\mathfrak{p}}+1-a_{\mathfrak{p}}\left(\mathcal{X}_{\mathfrak{p}, c}\right)
$$

et $m_{c}=1$. Supposons $c \in \Delta_{\mathfrak{p}}\left(\kappa_{\mathfrak{p}}\right)$. On peut passer en premier lieu à la structure réduite de $\mathcal{X}_{\mathfrak{p}, c}$ : en effet cela ne change ni le décompte des points rationnels, ni la cohomologie. Par la formule de Lefschetz ( $c f$. [5, Rapport, cor. 5.4] et [4, (1.5)], pour tout $c \in C_{\mathfrak{p}}\left(\kappa_{\mathfrak{p}}\right)$ on a

$$
\# \mathcal{X}_{\mathfrak{p}, c}\left(\kappa_{\mathfrak{p}}\right)=\sum_{i=0}^{2}(-1)^{i} \operatorname{Tr}\left(\bar{F}_{\mathfrak{p}} \mid H_{\underline{c}}^{i}\left(\overline{\mathcal{X}}_{\mathfrak{p}, c}, \mathbb{Q}_{\ell}\right)\right) \text {. }
$$

Ici $H_{\underline{c}}^{i}$ désigne la cohomologie $\ell$-adique à support propre. Comme $\mathcal{X}_{\mathfrak{p}, c}$ est connexe, on a

$$
\operatorname{Tr}\left(\bar{F}_{\mathfrak{p}} \mid H_{\underline{c}}^{0}\left(\overline{\mathcal{X}}_{\mathfrak{p}, c}, \mathbb{Q}_{\ell}\right)\right)=1 \text {. }
$$

De plus, par définition, $a_{\mathfrak{p}}\left(\mathcal{X}_{\mathfrak{p}, c}\right)=\operatorname{Tr}\left(\bar{F}_{\mathfrak{p}} \mid H_{\underline{c}}^{1}\left(\overline{\mathcal{X}}_{\mathfrak{p}, c}, \mathbb{Q}_{\ell}\right)\right)$.

Soit $\vartheta_{\mathfrak{p}}: \mathcal{C}_{\mathfrak{p}} \rightarrow \overline{\mathcal{X}}_{\mathfrak{p}, c}$ une normalisation de $\overline{\overline{\mathcal{X}}}_{\mathfrak{p}, c}$. La courbe $\mathcal{C}_{\mathfrak{p}}$ est une réunion disjointe de courbes projectives lisses. Le morphisme $\vartheta_{\mathfrak{p}}$ induit une suite spectrale de Leray $E^{r, s}:=H_{\underline{c}}^{r}\left(\overline{\mathcal{X}}_{\mathfrak{p}, c}, R^{s}\left(\vartheta_{\mathfrak{p}}\right)_{*} \mathbb{Q}_{\ell}\right) \Rightarrow E^{r+s}:=H_{\underline{c}}^{r+s}\left(\mathcal{C}_{\mathfrak{p}}, \mathbb{Q}_{\ell}\right)$. Elle 
induit la suite exacte

$$
\begin{aligned}
& 0 \rightarrow H_{\underline{c}}^{1}\left(\overline{\mathcal{X}}_{\mathfrak{p}, c},\left(\vartheta_{\mathfrak{p}}\right)_{*} \mathbb{Q}_{\ell}\right) \longrightarrow H_{\underline{c}}^{1}\left(\mathcal{C}_{\mathfrak{p}}, \mathbb{Q}_{\ell}\right) \longrightarrow H_{\underline{c}}^{0}\left(\overline{\mathcal{X}}_{\mathfrak{p}, c}, R_{c}^{1}\left(\left(\vartheta_{\mathfrak{p}}\right)_{*} \mathbb{Q}_{\ell}\right)\right) \\
& \quad \longrightarrow H_{\underline{c}}^{2}\left(\overline{\mathcal{X}}_{\mathfrak{p}, c},\left(\vartheta_{\mathfrak{p}}\right)_{*} \mathbb{Q}_{\ell}\right) \longrightarrow H_{\underline{c}}^{2}\left(\mathcal{C}_{\mathfrak{p}}, \mathbb{Q}_{\ell}\right) \longrightarrow H_{\underline{c}}^{1}\left(\overline{\mathcal{X}}_{\mathfrak{p}, c}, R_{\underline{c}}^{1}\left(\left(\vartheta_{\mathfrak{p}}\right)_{*} \mathbb{Q}_{\ell}\right)\right)
\end{aligned}
$$

Il suit de [9, th. 3.2 (b), ch. VI, p. 228] que pour tout $i>0$ on a $R_{\underline{c}}^{i}\left(\vartheta_{\mathfrak{p}}\right)_{*}=0$, donc $H_{\underline{c}}^{2}\left(\mathcal{C}_{\mathfrak{p}}, \mathbb{Q}_{\ell}\right) \cong H_{\underline{c}}^{2}\left(\overline{\mathcal{X}}_{\mathfrak{p}, c},\left(\vartheta_{\mathfrak{p}}\right)_{*} \mathbb{Q}_{\ell}\right)$. De plus, le premier groupe est une somme directe de $\mathbb{Q}_{\ell}(-1)$. Pour chaque composante $\kappa_{\mathfrak{p}}$-rationnelle de $\mathcal{C}_{\mathfrak{p}}$, l'automorphisme de Frobenius $\bar{F}_{\mathfrak{p}}$ agit sur la composante correspondante dans $H_{\underline{c}}^{2}\left(\mathcal{C}_{\mathfrak{p}}, \mathbb{Q}_{\ell}\right)$ par multiplication par $q_{\mathfrak{p}}$. Les autres composantes de $\mathcal{C}_{\mathfrak{p}}$ sont permutées entre elles, a fortiori la trace de $\bar{F}_{\mathfrak{p}}$ dans les composantes correspondantes dans $H_{c}^{2}\left(\mathcal{C}_{\mathfrak{p}}, \mathbb{Q}_{\ell}\right)$ est égale à 0 . D'autre part, les valeurs propres de $\bar{F}_{\mathfrak{p}}$ dans $H_{\underline{c}}^{2}\left(\overline{\overline{\mathcal{X}}}_{\mathfrak{p}, c},\left(\vartheta_{\mathfrak{p}}\right)_{*} \mathbb{Q}_{\ell}\right)$ sont égales aux valeurs propres de $\bar{F}_{\mathfrak{p}}$ sur $H_{\underline{c}}^{2}\left(\overline{\mathcal{X}}_{\mathfrak{p}, c}, \mathbb{Q}_{\ell}\right)$. D'où $\operatorname{Tr}\left(\bar{F}_{\mathfrak{p}} \mid H_{\underline{c}}^{2}\left(\overline{\mathcal{X}}_{\mathfrak{p}, c}, \mathbb{Q}_{\ell}\right)\right)=q_{\mathfrak{p}} m_{c}$ et cela achève la preuve du lemme.

Nous passons maintenant à l'étude de la $K / k$-trace notée $B$.

Soit $\operatorname{Alb}(\mathcal{X})$, respectivement $\operatorname{Alb}(C)$, la variété Albanese de $\mathcal{X}$, respectivement de $C$. Soit $(\tau, B)$ la $K / k$-trace de $J_{X}$. Soient $\operatorname{Pic}^{0}(C)$ le groupe des diviseurs algébriquement équivalents à zéro de $C$ et $f^{*}: \operatorname{Pic}^{0}(C) \rightarrow \operatorname{Pic}^{0}(\mathcal{X})$ l'application «pull-back» déduite de $f$.

Soient $\imath: X \rightarrow \mathcal{X}$ l'inclusion de la fibre générique et $\imath^{*}: \operatorname{Div}(\mathcal{X}) \rightarrow \operatorname{Div}(X)$ la restriction des diviseurs à la fibre générique $X$. Par construction de la $K / k$ trace, l'homomorphisme induit de $\operatorname{Pic}^{0}(\mathcal{X})$ vers $\operatorname{Pic}^{0}(X)$ se factorise par un homomorphisme $b: \operatorname{Pic}^{0}(\mathcal{X}) \rightarrow B$.

Proposition 3.3. - Le groupe $\operatorname{Ker}\left(f^{*}\right)$ est fini et est même trivial si $f$ admet une section. La suite de variétés abéliennes

$$
0 \rightarrow \operatorname{Pic}^{0}(C) \stackrel{f^{*}}{\longrightarrow} \operatorname{Pic}^{0}(\mathcal{X}) \stackrel{b}{\longrightarrow} B \rightarrow 0
$$

est exacte si $f$ admet une section et exacte à des groupes finis près en général. En particulier, $H_{\text {êt }}^{1}\left(\overline{\mathcal{X}}, \mathbb{Q}_{\ell}\right) \cong H_{\text {ét }}^{1}\left(\bar{C}, \mathbb{Q}_{\ell}\right) \oplus H_{\text {ét }}^{1}\left(\bar{B}, \mathbb{Q}_{\ell}\right)$. A fortiori, pour presque tout idéal premier $\mathfrak{p}$ de $\mathcal{O}_{k}$, on a $a_{\mathfrak{p}}(\mathcal{X})=a_{\mathfrak{p}}(C)+a_{\mathfrak{p}}(B)$.

Remarque 3.4. - La formule $B=\operatorname{Pic}^{0}(\mathcal{X}) / f^{*} \operatorname{Pic}^{0}(C)$ est donnée (sans preuve) dans [17] et l'exactitude de la suite est démontrée par Shioda dans [15] sous l'hypothèse d'existence d'une section mais en complète généralité concernant la caractéristique et la structure de schéma (le résultat est attribué à Raynaud).

Démonstration. - Il est clair que $\operatorname{Im}\left(f^{*}\right) \subset \operatorname{Ker}(b)$, car $b \circ f^{*}=0$. Si $D$ est un diviseur sur $\mathcal{X}$ dont la classe est dans $\operatorname{Ker}(b)$, on voit que $D=\operatorname{div}(g)+V$, où $\operatorname{div}(g)$ est le diviseur d'une fonction rationnelle $g \in \bar{k}(\mathcal{X})$ et $V$ est un diviseur 
vertical, i.e., à support dans les fibres de $f$. Mais $D$ et donc $V$ sont algébriquement équivalents à zéro, et donc numériquement triviaux, donc, d'après le Lemme 3.1, $V$ est une somme de fibres, c'est-à-dire qu'il est de la forme $f^{*}\left(D^{\prime}\right)$. A priori on doit prendre $D^{\prime}$ dans $\operatorname{Pic}^{0}(C) \otimes \mathbb{Q}$ et donc il existe $m \geq 1$ tel que $m V \in f^{*} \operatorname{Pic}^{0}(C)$, mais si $f$ admet une section, il n'y a pas de fibres multiples et on peut prendre $m=1$; en général $m$ est borné par la multiplicité des fibres. On a donc bien $\operatorname{Im}\left(f^{*}\right)=\operatorname{Ker}(b)$.

Soit $C_{0} \subset \mathcal{X}$ une courbe irréductible fermée telle que $f_{\mid C_{0}}: C_{0} \rightarrow C$ soit dominante, donc un morphisme fini de degré disons $d$. Notons $j$ l'inclusion $C_{0} \hookrightarrow \mathcal{X}$, donc $f_{\mid C_{0}}=f^{\prime}=f \circ j$. L'hypothèse $f^{*}(z)=0$ entraîne $f^{\prime *}(z)=0$ donc $d z=f_{*}^{\prime} f^{\prime *}(z)=0$. Ainsi $\operatorname{Ker}\left(f^{*}\right) \subset \operatorname{Pic}^{0}(C)[d]$ est fini (et même trivial si on peut choisir $d=1$, i.e., si $C_{0}$ est une section de $f$ ).

Pour prouver que $b$ est surjective, il suffit de prouver que

$$
\operatorname{dim}(B) \leq \operatorname{dim}\left(\operatorname{Pic}^{0}(\mathcal{X})\right)-\operatorname{dim}\left(\operatorname{Pic}^{0}(C)\right),
$$

ou encore que $\operatorname{dim}(\operatorname{Alb}(C))+\operatorname{dim}(B) \leq \operatorname{dim}(\operatorname{Alb}(\mathcal{X}))$ (il y aura alors égalité). L'homomorphisme $\tau: B \rightarrow J_{X}$ est injectif, parce que $k$ est un corps de nombres. Soit $\tau^{\vee}: J_{X} \rightarrow B^{\vee}$ son homomorphisme dual; c'est en fait la $K / k$-image de $J_{X}, c f$. [8, ch. VIII]. Notons que par extension des scalaires, et en tenant compte que $\bar{K}$ contient $\bar{k}(C)$, la $K / k$-trace et la $K / k$-image de $J_{X}$ coïcident avec la $\bar{K} / \bar{k}$-trace et la $\bar{K} / \bar{k}$-image de $J_{X}$. Choisissons un morphisme fini $X \rightarrow J_{X}$ défini sur $K$ et dont l'image engendre $J_{X}$. En composant avec $\tau$, on obtient une application qui se prolonge en une application rationnelle $\mathcal{X} \rightarrow B^{\vee} \times C$ définie sur $k$. En composant avec l'application canonique $\pi_{C}: C \rightarrow \operatorname{Alb}(C)$, on obtient une application, a priori rationnelle $\alpha: \mathcal{X} \rightarrow B^{\vee} \times \operatorname{Alb}(C)$ qui doit en fait être un morphisme, puisque $\mathcal{X}$ est lisse (cf. [8, ch. II, th. 1]). Ce dernier morphisme induit donc, par la propriété universelle de la variété d'Albanese, un homomorphisme $\widehat{\alpha}: \operatorname{Alb}(\mathcal{X}) \rightarrow B^{\vee} \times \operatorname{Alb}(C)$ dont on montre qu'il est surjectif, ce qui achève la démonstration de l'exactitude. Pour vérifier que $\widehat{\alpha}$ est surjective (donc c'est une isogénie), il faut montrer que $\alpha(\mathcal{X})$ engendre la variété abélienne $B^{\vee} \times \operatorname{Alb}(C)$. L'image de la fibre générique $X$ dans sa jacobienne $J_{X}$ engendre $J_{X}$, donc son image par $\tau^{\vee}$ engendre $B^{\vee}$ et comme l'image $\pi_{C}(C)$ engendre $\operatorname{Alb}(C)$, on peut conclure.

Pour l'isomorphisme entre les groupes de cohomologie étale, comme $\operatorname{Ker}\left(f^{*}\right)$ est fini, la tensorisation par $\mathbb{Q}_{\ell}$ donne $H_{\text {ét }}^{1}\left(\operatorname{Pic}^{0}(C), \mathbb{Q}_{\ell}\right) \cong H_{\text {ét }}^{1}\left(\bar{C}, \mathbb{Q}_{\ell}\right)$, donc l'affirmation sur la trace est immédiate.

Nous passons maintenant à la formule de Shioda-Tate.

L'ensemble $I_{X}=\{(D \cdot X) \mid D \in \operatorname{Div}(\mathcal{X})\}$ est un idéal de $\mathbb{Z}$. Soit $\left(D_{0} \cdot X\right)$ un générateur de $I_{X}$, où $D_{0}$ est un diviseur sur $\mathcal{X}$. Soient $\imath: X \rightarrow \mathcal{X}$ l'inclusion de la fibre générique et $\imath^{*}: \operatorname{Div}(\mathcal{X}) \rightarrow \operatorname{Div}(X)$ la restriction des diviseurs à la 
fibre générique $X$. Définissons $\psi: \operatorname{Pic}(\mathcal{X}) \rightarrow J_{X}$ par

$$
\psi(\operatorname{cl}(D))=\imath^{*}\left(D-\frac{(D \cdot X)}{\left(D_{0} \cdot X\right)} D_{0}\right) .
$$

On obtient alors d'après le lemme ci-dessous (3.7) une suite exacte

$$
0 \rightarrow \operatorname{Ker}(\psi) \longrightarrow \operatorname{Pic}(\mathcal{X}) \stackrel{\psi}{\longrightarrow} J_{X} \rightarrow 0 .
$$

DÉfinition 3.5. - Soit $\widetilde{\mathcal{S}}$ (respectivement $\mathcal{S}$ ) le sous-groupe $\operatorname{de} \operatorname{Pic}(\mathcal{X})$ (resp. de $\operatorname{NS}(\mathcal{X})$ ) engendré par les classes de $D_{0}$ et des composantes des fibres de $f$. On notera respectivement $\widetilde{\mathcal{S}}_{\mathbb{Q}}$ et $\mathcal{S}_{\mathbb{Q}}$ les espaces vectoriels obtenus par tensorisation par $\mathbb{Q}$.

Remarque 3.6. - Soit $\mathfrak{p} \notin S$ et $X_{\mathfrak{p}}$ la fibre générique de $f_{\mathfrak{p}}$. L'ensemble $I_{X_{\mathfrak{p}}}:=\left\{\left(D \cdot X_{\mathfrak{p}}\right) \mid D \in \operatorname{Div}\left(\mathcal{X}_{\mathfrak{p}}\right)\right\}$ est un idéal de $\mathbb{Z}$. Soit $\mathcal{D}_{1, \mathfrak{p}}$ un diviseur de $\mathcal{X}_{\mathfrak{p}}$ tel que $\left(\mathcal{D}_{1, \mathfrak{p}} \cdot X_{\mathfrak{p}}\right)$ engendre $I_{X_{\mathfrak{p}}}$. Soit $\mathcal{S}_{\mathfrak{p}}$ le sous-espace de $\operatorname{NS}\left(\mathcal{X}_{\mathfrak{p}}\right)$ engendré par les classes de $\mathcal{D}_{1, \mathfrak{p}}$ et des composantes des fibres de $f_{\mathfrak{p}}$. Pour tout $\mathfrak{p}$ (sauf un nombre fini), $\mathcal{S}_{\mathfrak{p}}$ coïncide avec la réduction de $\mathcal{S}$ modulo $\mathfrak{p}$. Donc, après avoir élargi si nécessaire $S$, on peut supposer cela vrai pour tout $\mathfrak{p}$ hors de $S$. En particulier, pour $\mathfrak{p}$ hors de $S$, on aura $\operatorname{Tr}\left(F_{\mathfrak{p}} \mid \mathcal{S}^{I_{\mathfrak{p}}}\right)=\operatorname{Tr}\left(\bar{F}_{\mathfrak{p}} \mid \mathcal{S}_{\mathfrak{p}}\right)$.

LEMME 3.7. - L'application $\psi$ est surjective et son noyau $\operatorname{Ker}(\psi)$ est égal $\grave{a} \widetilde{\mathcal{S}}$. Une base de $\mathcal{S}_{\mathbb{Q}}$ est fournie par les classes de $D_{0}$, d'une fibre lisse $\mathcal{F}$ de $f$ et par les composantes, sauf une, de chaque fibre singulière non irréductible $f^{-1}(c)=\mathcal{X}_{c}$ de $f$. Soit $n_{c}$ le nombre des composantes de $\mathcal{X}_{c}$. On a en particulier,

$$
\operatorname{rang}(\mathcal{S})=2+\sum_{c \in C}\left(n_{c}-1\right) .
$$

Démonstration. - Soit $P \in J_{X}$ tel que $P$ représente la classe $\operatorname{cl}(\mathcal{D})$ d'un diviseur $\mathcal{D} \in \operatorname{Div}(X)$. Soit $\overline{\mathcal{D}}$ la clôture de Zariski de $\mathcal{D}$ dans $\mathcal{X}$. C'est un diviseur de Weil (donc de Cartier, puisque $\mathcal{X}$ est lisse) et il est immédiat qu'on a $\psi(\operatorname{cl}(\overline{\mathcal{D}}))=P$, où $\operatorname{cl}(\overline{\mathcal{D}})$ dénote la classe de $\overline{\mathcal{D}}$. Ainsi $\psi$ est bien surjective.

Il est clair que $\widetilde{\mathcal{S}} \subset \operatorname{Ker}(\psi)$. Supposons que $\operatorname{cl}(D) \in \operatorname{Ker}(\psi)$. Alors, il existe une fonction $x \in \bar{K}(X)$ telle que $\operatorname{div}(x)=\imath^{*}\left(D-\left((D \cdot X) /\left(D_{0} \cdot X\right)\right) D_{0}\right)$. Soit $\widetilde{x}$ une fonction rationnelle sur $\mathcal{X}$ telle que $\widetilde{x}_{\mid X}=x$. En particulier, $\imath^{*} \operatorname{div}(\tilde{x})=$ $\operatorname{div}(x)$. Il est clair que, au niveau des diviseurs, $\operatorname{Ker}\left(\imath^{*}\right)$ est engendré par les classes des composantes irréductibles des fibres $\mathcal{X}_{c}$ de $f$. Par conséquent $\operatorname{div}(\widetilde{x})-\left(D-\left((D \cdot X) /\left(D_{0} \cdot X\right)\right) D_{0}\right)$ est une somme des composantes de ces fibres. On en tire bien que $\operatorname{cl}(D) \in \widetilde{\mathcal{S}}$ et, par suite $\operatorname{Ker}(\psi)=\widetilde{\mathcal{S}}$.

Pour la dernière affirmation, il est clair que les classes de l'énoncé forment un système générateur car toutes les fibres sont algébriquement équivalentes. Le fait qu'elles soient indépendantes se vérifie en utilisant la théorie d'intersection et le lemme 3.1. La formule donnant le rang de $\mathcal{S}$ est alors immédiate. 
Proposition 3.8 (formule de Shioda-Tate). - Il existe un isomorphisme de $\mathbb{Q}\left[G_{k}\right]$-modules

$$
\mathrm{NS}(\mathcal{X}) \otimes \mathbb{Q} \cong\left(\left(\frac{J_{X}(\bar{k}(C))}{\tau B(\bar{k})}\right) \otimes \mathbb{Q}\right) \oplus \mathcal{S}_{\mathbb{Q}}
$$

En particulier, on a

$$
\operatorname{rang}(\mathrm{NS}(\mathcal{X} / k))=\operatorname{rang}\left(\frac{J_{X}(K)}{\tau B(k)}\right)+\operatorname{rang}\left(\mathcal{S}^{G_{k}}\right) .
$$

Démonstration. - Remarquons que, si l'on travaille sur les $\mathbb{Q}$-espaces vectoriels, on peut supposer que $D_{0}$ soit défini sur $k$ (la seule différence est que $(D \cdot X) /\left(D_{0} \cdot X\right)$ est maintenant un nombre rationnel). Les suites exactes (3.1) et $(3.2)$ et le fait que $\operatorname{Ker}(\psi)=\widetilde{\mathcal{S}}$ nous donnent une suite exacte et scindée après tensorisation par $\mathbb{Q}$

$$
0 \rightarrow \mathcal{S} \rightarrow \mathrm{NS}(\mathcal{X}) \stackrel{\vartheta}{\longrightarrow} \frac{J_{X}(\bar{k}(C))}{\tau B(\bar{k})} \rightarrow 0,
$$

le résultat en découle.

\section{Preuve du théorème 1.3}

Soient $\mathcal{S}_{\ell}(1)=\mathcal{S} \otimes T_{\ell}(\bar{k})$ le «twist » à la Tate du $G_{k}$-module $\mathcal{S}$ et $L\left(\mathcal{S}_{\ell}(1), s\right)$ la fonction $L$ d'Artin associée à la représentation donnée par $\mathcal{S}_{\ell}(1)$. Comme dans [14], la proposition 3.8 nous suggère de considérer la fonction

$$
N(\mathcal{X} / k, s)=L_{2}(\mathcal{X} / k, s) / L\left(\mathcal{S}_{\ell}(1), s\right) .
$$

Proposition 4.1. - Avec les notations précédentes, on a

$$
\operatorname{rang}\left(\frac{J_{X}(K)}{\tau B(k)}\right)+\underset{s=2}{\operatorname{ord}}(N(\mathcal{X} / k, s))=\operatorname{rang}(\mathrm{NS}(\mathcal{X} / k))+\underset{s=2}{\operatorname{ord}}\left(L_{2}(\mathcal{X} / k, s)\right) .
$$

Démonstration. - D'après la proposition 3.8 et la définition de $N(\mathcal{X} / k, s)$, il suffit de prouver que

$$
-\underset{s=2}{\operatorname{ord}}\left(L\left(\mathcal{S}_{\ell}(1), s\right)\right)=\operatorname{rang}\left(\mathcal{S}^{G_{k}}\right) .
$$

Cela est une conséquence du résultat suivant.

Proposition 4.2 (Artin, Brauer, [14, prop. 1.5.1]). - Soit $V$ un $\mathbb{Q}$-espace vectoriel de dimension finie avec une action continue de $G_{k}$, et soit $L(V, s)$ la fonction $L$ d'Artin associée.

1) $L(V, s)$ a une continuation méromorphe à $\mathbb{C}$.

2) $L(V, s)$ est holomorphe sur la droite $\operatorname{Re}(s)=1$ à l'exception éventuelle du point $s=1$, où l'on $a \operatorname{ord}_{s=1}(L(V, s))=-\operatorname{dim}\left(V^{G_{k}}\right)$.

3) $L(V, s)$ ne s'annule pas sur la droite $\operatorname{Re}(s)=1$. 
Proposition 4.3. - On a la formule

$$
\frac{\mathrm{d}}{\mathrm{d} s}(\log (N(\mathcal{X} / k, s)))=\sum_{\mathfrak{p} \notin S} A_{\mathfrak{p}}^{*}(\mathcal{X}) \frac{\log \left(q_{\mathfrak{p}}\right)}{q_{\mathfrak{p}}^{s-1}}+h(s),
$$

avec $h(s)$ fonction holomorphe sur le demi-plan $\operatorname{Re}(s)>\frac{3}{2}$.

Démonstration. - La fonction-zêta de $\mathcal{X}$ s'écrit comme

$$
\zeta(\mathcal{X} / k, s)=\prod_{i=0}^{4} P_{i}(s)^{(-1)^{i+1}}
$$

où $P_{i}(s)=\prod_{\mathfrak{p}} \operatorname{det}\left(1-F_{\mathfrak{p}} q_{\mathfrak{p}}^{-s} \mid H_{\text {ét }}^{i}\left(\overline{\mathcal{X}}, \mathbb{Q}_{\ell}\right)^{I_{\mathfrak{p}}}\right)^{-1}$. Notons que $P_{0}(s)=\zeta_{k}(s)$ et $P_{4}(s)=\zeta_{k}(s+2)$. En plus $P_{3}(s)=P_{1}(s+1)[13,5.6]$. La formule de Lefschetz et la proposition 3.3 impliquent

$$
\# \mathcal{X}_{\mathfrak{p}}\left(\kappa_{\mathfrak{p}}\right)=1-a_{\mathfrak{p}}(C)-a_{\mathfrak{p}}(B)+b_{\mathfrak{p}}(\mathcal{X})-q_{\mathfrak{p}} a_{\mathfrak{p}}(C)-q_{\mathfrak{p}} a_{\mathfrak{p}}(B)+q_{\mathfrak{p}}^{2},
$$

où $b_{\mathfrak{p}}(\mathcal{X})$ dénote la trace de $F_{\mathfrak{p}}$ dans $H_{\text {ét }}^{2}\left(\overline{\mathcal{X}}, \mathbb{Q}_{\ell}\right)^{I_{\mathfrak{p}}}$. D'autre part, par le lemme 3.2 on a

$$
\begin{aligned}
\# \mathcal{X}_{\mathfrak{p}}\left(\kappa_{\mathfrak{p}}\right) & =\sum_{c \in C_{\mathfrak{p}}\left(\kappa_{\mathfrak{p}}\right)} \# \mathcal{X}_{\mathfrak{p}, c}\left(\kappa_{\mathfrak{p}}\right) \\
& =\sum_{c \in C_{\mathfrak{p}}\left(\kappa_{\mathfrak{p}}\right)}\left(q_{\mathfrak{p}}+1+\left(m_{c}-1\right) q_{\mathfrak{p}}-a_{\mathfrak{p}}\left(\mathcal{X}_{\mathfrak{p}, c}\right)\right) \\
& =\left(q_{\mathfrak{p}}+1\right) \# C_{\mathfrak{p}}\left(\kappa_{\mathfrak{p}}\right)-q_{\mathfrak{p}} A_{\mathfrak{p}}(\mathcal{X})+\left(\sum_{c \in C_{\mathfrak{p}}\left(\kappa_{\mathfrak{p}}\right)}\left(m_{c}-1\right)\right) q_{\mathfrak{p}}
\end{aligned}
$$

Par ailleurs $\# C_{\mathfrak{p}}\left(\kappa_{\mathfrak{p}}\right)=q_{\mathfrak{p}}+1-a_{\mathfrak{p}}(C)$. En tenant compte aussi que $a_{\mathfrak{p}}(B)=$ $O\left(q_{\mathfrak{p}}{ }^{1 / 2}\right)$, on obtient de (4.3) et (4.4) que

$$
q_{\mathfrak{p}} A_{\mathfrak{p}}^{*}(\mathcal{X})=-b_{\mathfrak{p}}(\mathcal{X})+2 q_{\mathfrak{p}}+\left(\sum_{c \in C_{\mathfrak{p}}\left(\kappa_{\mathfrak{p}}\right)}\left(m_{c}-1\right)\right) q_{\mathfrak{p}}+O\left(q_{\mathfrak{p}}^{1 / 2}\right) .
$$

Le Frobenius $\bar{F}_{\mathfrak{p}}$ fixe les classes de $D_{0}(\bmod \mathfrak{p})$ et $\mathcal{F}(\bmod \mathfrak{p})$ et permute les composantes des fibres singulières de $f_{\mathfrak{p}}$. Donc, la trace de $\bar{F}_{\mathfrak{p}}$ sur $\mathcal{S}_{\mathfrak{p}}$ est égale à 2 plus le nombre des composantes $\kappa_{\mathfrak{p}}$-rationnelles, i.e.,

$$
\operatorname{Tr}\left(\bar{F}_{\mathfrak{p}} \mid \mathcal{S}_{\mathfrak{p}}\right)=\operatorname{Tr}\left(F_{\mathfrak{p}} \mid \mathcal{S}^{I_{\mathfrak{p}}}\right)=2+\sum_{c \in C_{\mathfrak{p}}\left(\kappa_{\mathfrak{p}}\right)}\left(m_{c}-1\right) .
$$

Il suit donc de (4.5) et (4.6) que

$$
A_{\mathfrak{p}}^{*}(\mathcal{X})=-\frac{b_{\mathfrak{p}}(\mathcal{X})}{q_{\mathfrak{p}}}+\operatorname{Tr}\left(F_{\mathfrak{p}} \mid \mathcal{S}^{I_{\mathfrak{p}}}\right)+O\left(q_{\mathfrak{p}}^{-1 / 2}\right) .
$$

BULletin DE LA SOCiÉtÉ MATHÉMATIQUe DE FRANCE 
On calcule maintenant les dérivées logarithmiques de $L\left(\mathcal{S}_{\ell}(1), s\right)$ et de $L_{2}(\mathcal{X} / k, s)$. Pour la première on a

$$
\begin{gathered}
\frac{\mathrm{d}}{\mathrm{d} s}\left(\log \left(L\left(\mathcal{S}_{\ell}(1), s\right)\right)\right)=\frac{\mathrm{d}}{\mathrm{d} s}\left(\sum_{\mathfrak{p}}-\log \left(\operatorname{det}\left(1-F_{\mathfrak{p}} q_{\mathfrak{p}}^{-s} \mid \mathcal{S}_{\ell}(1)\right)^{I_{\mathfrak{p}}}\right)\right) \\
=\sum_{\mathfrak{p} \notin S}-\operatorname{Tr}\left(F_{\mathfrak{p}} \mid \mathcal{S}\right) \frac{\log \left(q_{\mathfrak{p}}\right)}{q_{\mathfrak{p}}^{s-1}}+f_{1}(s),
\end{gathered}
$$

avec $f_{1}(s)$ holomorphe sur le demi-plan $\operatorname{Re}(s)>\frac{3}{2}$. Pour la deuxième,

$$
\begin{aligned}
\frac{\mathrm{d}}{\mathrm{d} s}\left(\log \left(L_{2}(\mathcal{X} / k, s)\right)\right) & =\frac{\mathrm{d}}{\mathrm{d} s}\left(\sum_{\mathfrak{p}}-\log \left(\operatorname{det}\left(1-F_{\mathfrak{p}} q_{\mathfrak{p}}^{-s} \mid H_{\text {ét }}^{2}\left(\overline{\mathcal{X}}, \mathbb{Q}_{\ell}\right)\right)^{I_{\mathfrak{p}}}\right)\right) \\
& =\sum_{\mathfrak{p} \notin S}-b_{\mathfrak{p}}(\mathcal{X}) \frac{\log \left(q_{\mathfrak{p}}\right)}{q_{\mathfrak{p}}^{s}}+f_{2}(s)
\end{aligned}
$$

avec une fonction $f_{2}(s)$ holomorphe pour $\operatorname{Re}(s)>\frac{3}{2}$. En effet, pour ce demi-plan les conjectures de Weil (Deligne) nous donnent $\left|b_{\mathfrak{p}}(\mathcal{X})\right| \leq q_{\mathfrak{p}} \operatorname{dim}\left(H_{\text {ét }}^{2}\left(\overline{\mathcal{X}}, \mathbb{Q}_{\ell}\right)\right)$.

Finalement, par la définition de $N(\mathcal{X} / k, s),(4.8)$ et (4.9) on obtient

$$
\begin{aligned}
\frac{\mathrm{d}}{\mathrm{d} s}(\log (N(\mathcal{X} / k, s))) & =\frac{\mathrm{d}}{\mathrm{d} s}\left(\log \left(L_{2}(\mathcal{X} / k, s)\right)\right)-\frac{\mathrm{d}}{\mathrm{d} s}\left(\log \left(L\left(\mathcal{S}_{\ell}(1), s\right)\right)\right) \\
& =\sum_{\mathfrak{p} \notin S}-b_{\mathfrak{p}}(\mathcal{X}) \frac{\log \left(q_{\mathfrak{p}}\right)}{q_{\mathfrak{p}}^{s}}-\sum_{\mathfrak{p} \notin S}-\operatorname{Tr}\left(F_{\mathfrak{p}} \mid \mathcal{S}\right) \frac{\log \left(q_{\mathfrak{p}}\right)}{q_{\mathfrak{p}}^{s-1}}+h_{1}(s) \\
& =\sum_{\mathfrak{p}} A_{\mathfrak{p}}^{*}(\mathcal{X}) \frac{\log \left(q_{\mathfrak{p}}\right)}{q_{\mathfrak{p}}^{s-1}}+h_{2}(s),
\end{aligned}
$$

avec $h_{2}(s)$ holomorphe pour $\operatorname{Re}(s)>\frac{3}{2}$ parce que la série $\sum_{\mathfrak{p} \notin S} q_{\mathfrak{p}}^{-1 / 2} \log \left(q_{\mathfrak{p}}\right) / q_{\mathfrak{p}}^{\sigma-1}$ est convergente pour $\sigma>\frac{3}{2}$, où $\operatorname{Re}(s)=\sigma$.

Démonstration du théorème 1.3. - Les propositions 4.1 et 4.3 et la conjecture 1.1 nous donnent

$$
\begin{gathered}
\operatorname{Rés}_{s=1}\left(\sum_{\mathfrak{p}}-A_{\mathfrak{p}}^{*}(\mathcal{X}) \frac{\log \left(q_{\mathfrak{p}}\right)}{q_{\mathfrak{p}}^{s}}\right)=-\operatorname{Rés}_{s=2}\left(\frac{\mathrm{d}}{\mathrm{d} s}(\log (N(\mathcal{X} / k, s)))\right)=-\underset{s=2}{\operatorname{ord}}(N(\mathcal{X} / k, s)) \\
=-\operatorname{rang}(\operatorname{NS}(\mathcal{X}))+\operatorname{rang}\left(\frac{J_{X}(K)}{\tau B(k)}\right)-\operatorname{ord}_{s=2} L_{2}(\mathcal{X}, s)=\operatorname{rang}\left(\frac{J_{X}(K)}{\tau B(k)}\right) .
\end{gathered}
$$

Par la proposition $4.2, L\left(\mathcal{S}_{\ell}(1), s\right)$ ne s'annule pas sur la droite $\operatorname{Re}(s)=2$. En supposant de plus que $L_{2}(\mathcal{X} / k, s)$ ne s'annule pas sur la droite précédente, 
on peut appliquer les théorèmes taubériens standard [7, ch. XV] obtenant

$$
\begin{aligned}
\lim _{T \rightarrow \infty} \frac{1}{T}\left(\sum_{\substack{\mathfrak{p} \notin S \\
q_{\mathfrak{p}} \leq T}}-b_{\mathfrak{p}}(\mathcal{X}) \frac{\log \left(q_{\mathfrak{p}}\right)}{q_{\mathfrak{p}}}\right) & =\operatorname{Rés}_{s=2}\left(\frac{\mathrm{d}}{\mathrm{d} s}\left(\log \left(L_{2}(\mathcal{X} / k, s)\right)\right)\right) \\
& =\underset{s=2}{\operatorname{ord}}\left(L_{2}(\mathcal{X} / k, s)\right) \\
\lim _{T \rightarrow \infty} \frac{1}{T}\left(\sum_{\substack{\mathfrak{p} \notin S \\
q_{\mathfrak{p}} \leq T}}-\operatorname{Tr}\left(F_{\mathfrak{p}} \mid \mathcal{S}\right) \log \left(q_{\mathfrak{p}}\right)\right) & =\operatorname{Rés}_{s=2}\left(\frac{\mathrm{d}}{\mathrm{d} s}\left(\log \left(L\left(\mathcal{S}_{\ell}(1), s\right)\right)\right)\right) \\
& =\operatorname{ord}\left(L\left(\mathcal{S}_{\ell}(1), s\right)\right) .
\end{aligned}
$$

En utilisant ces dernières formules, la proposition 4.1 et la conjecture 1.1 , on peut conclure

$$
\begin{aligned}
\lim _{T \rightarrow \infty} \frac{1}{T}\left(\sum_{\substack{\mathfrak{p} \notin S \\
q_{\mathfrak{p}} \leq T}}-A_{\mathfrak{p}}^{*}(\mathcal{X}) \log \left(q_{\mathfrak{p}}\right)\right) & =-\underset{s=2}{\operatorname{ord}}\left(L_{2}(\mathcal{X} / k, s)\right)+\underset{s=2}{\operatorname{ord}}\left(L\left(\mathcal{S}_{\ell}(1), s\right)\right) \\
& =-\operatorname{ord}_{s=2}(N(\mathcal{X} / k, s))=\operatorname{rang}\left(\frac{J_{X}(K)}{\tau B(k)}\right)
\end{aligned}
$$

\section{Exemples}

Les exemples d'application potentielle du résultat sont très nombreux à cause de la proposition classique suivante.

Proposition 5.1. - Soit $\mathcal{X}$ une variété lisse et projective de dimension $n$, définie sur un corps infini $k$. Quitte à remplacer $\mathcal{X}$ par son éclatement en un nombre fini de points, il existe une fibration en courbes de genre $g \geq 1$, disons $f: \mathcal{X} \rightarrow \mathbb{P}^{n-1}$, également définie sur $k$.

Démonstration. - Pour le cas des surfaces, la construction d'un pinceau de Lefschetz répond à la question (voir par exemple [9, ch. V, prop. 3.1]). Dans le cas général où $\operatorname{dim}(\mathcal{X})=n$, prenons un plongement $\mathcal{X} \subset \mathbb{P}^{N}$ et une projection linéaire (à la Noether) induisant un morphisme fini $\phi: \mathcal{X} \rightarrow \mathbb{P}^{n}$. Soit $p_{0}$ un point de $\mathbb{P}^{n}$ situé hors du diviseur de ramification de $\phi$; soient $\pi: \widetilde{\mathbb{P}} \rightarrow \mathbb{P}^{n}$ l'éclatement du point $p_{0}$ et $\pi^{\prime}: \widetilde{\mathbb{P}} \rightarrow \mathbb{P}^{n-1}$ l'application associée (c'est un fibré en droites projectives). Si $\widetilde{\mathcal{X}}$ est l'éclaté de $\mathcal{X}$ au-dessus des points de $\phi^{-1}\left(p_{0}\right)$, on obtient un morphisme fini $\widetilde{\phi}: \widetilde{\mathcal{X}} \rightarrow \widetilde{\mathbb{P}}$ qui, composé avec $\pi^{\prime}$, fournit la fibration en courbes cherchée $f=\pi^{\prime} \circ \widetilde{\phi}: \widetilde{\mathcal{X}} \rightarrow \mathbb{P}^{n-1}$.

REMARQUE 5.2. - En reprenant la construction précédente, on voit qu'on a éclaté $\mathcal{X}$ en $d$ points (avec $d:=\operatorname{deg}_{\mathbb{P}^{N}} \mathcal{X}$ ). Si l'on considère $E$ l'un des diviseurs exceptionnels sur $\widetilde{\mathcal{X}}$, il est isomorphe à $\mathbb{P}^{n-1}$ et le morphisme $f_{\mid E}: E \rightarrow \mathbb{P}^{n-1}$ est de degré 1 donc est un isomorphisme. La construction fournit donc $d$ sections de $f: \tilde{\mathcal{X}} \rightarrow \mathbb{P}^{n-1}$ (définies sur une extension finie de $k$ ). 
Pour illustrer notre résultat, il convient de choisir des exemples de surfaces $\mathcal{X}$ pour lesquelles la conjecture de Tate (conjecture 1.1) est démontrée. Commençons par la proposition suivante qui est bien connue (par exemple l'argument est donné dans [14, th. 1.8], pour $\mathcal{X}=\mathbb{P}^{2}$ ).

Proposition 5.3. - Soient $\mathcal{X}, \mathcal{X}^{\prime}$ deux surfaces lisses et projectives définies sur $k$. Si $\mathcal{X}$ et $\mathcal{X}^{\prime}$ sont $k$-birationnellement équivalente alors la conjecture de Tate est vraie pour $\mathcal{X} / k$ si et seulement si elle est vraie pour $\mathcal{X}^{\prime} / k$.

Démonstration. - En utilisant qu'une application birationnelle entre surfaces lisses est une composition de transformations monoïdales et de ses inverses [6, ch. $\mathrm{V}]$, on se ramène au cas où on a un morphisme $\pi: \mathcal{X}^{\prime} \rightarrow \mathcal{X}$ composé d'un nombre fini d'éclatements. Chacune de ces transformations rajoute un diviseur exceptionnel $E$ au groupe de Néron-Severi et au groupe de cohomologie, c'est-à-dire $\mathrm{NS}\left(\mathcal{X}^{\prime}\right) \cong \pi^{*}\left(\mathrm{NS}((\mathcal{X})) \oplus \mathbb{Z}[E]\left[3\right.\right.$, prop. II.3] et $\left.H_{\text {ét }}^{2}\left(\overline{\mathcal{X}}^{\prime}, \mathbb{Q}_{\ell}\right)\right) \cong$ $\pi^{*}\left(H_{\text {ét }}^{2}\left(\overline{\mathcal{X}}, \mathbb{Q}_{\ell}\right)\right) \oplus \mathbb{Q}_{\ell}[E][14$, p. 255]. Globalement, en notant $V$ le groupe des classes de diviseurs engendré par les diviseurs exceptionnels, on obtient donc

$$
\mathrm{NS}\left(\mathcal{X}^{\prime}\right) \cong \mathrm{NS}(\mathcal{X}) \oplus V, \quad H_{\text {ét }}^{2}\left(\overline{\mathcal{X}}^{\prime}, \mathbb{Q}_{\ell}(1)\right) \cong H_{\text {ét }}^{2}\left(\overline{\mathcal{X}}, \mathbb{Q}_{\ell}(1)\right) \oplus\left(V \otimes \mathbb{Q}_{\ell}(1)\right) .
$$

Le quotient $L_{2}\left(\mathcal{X}^{\prime} / k, s\right) / L_{2}(\mathcal{X} / k, s)=L(V, s-1)$ est donc une fonction $L$ d'Artin en $s=2$ (cf. proposition 4.2) d'ordre

$$
\begin{aligned}
(5.1)-\underset{s=2}{\operatorname{ord}}(L(V, s-1)) & =\operatorname{dim}\left(\left(V \otimes \mathbb{Q}_{\ell}(1)\right)^{G_{k}}\right) \\
& =\operatorname{dim}\left(H_{\text {èt }}^{2}\left(\overline{\mathcal{X}}^{\prime}, \mathbb{Q}_{\ell}(1)\right)^{G_{k}}\right)-\operatorname{dim}\left(H_{\text {èt }}^{2}\left(\overline{\mathcal{X}}, \mathbb{Q}_{\ell}(1)\right)^{G_{k}}\right) .
\end{aligned}
$$

D'autre part on a bien

$$
\operatorname{rang}\left(\mathrm{NS}\left(\mathcal{X}^{\prime} / k\right)\right)-\operatorname{rang}(\mathrm{NS}(\mathcal{X} / k))=\operatorname{dim}\left(\left(V \otimes \mathbb{Q}_{\ell}(1)\right)^{G_{k}}\right)
$$

d'où le résultat.

Dans tous les cas où elle est démontrée, la conjecture 1.1 peut en fait se décomposer en deux parties ( $c f$. [13], [14]) dont la conjonction entraîne visiblement le résultat cherché :

- Conjecture de Tate I : on a un isomorphisme

$$
\mathrm{NS}(\mathcal{X} / k) \otimes \mathbb{Q}_{\ell} \cong H_{\text {ét }}^{2}\left(\overline{\mathcal{X}}, \mathbb{Q}_{\ell}(1)\right)^{G_{k}} .
$$

- Conjecture de Tate II : on a l'égalité

$$
-\underset{s=2}{\operatorname{ord}} L_{2}(\mathcal{X} / k, s)=\operatorname{dim} H_{\text {êt }}^{2}\left(\overline{\mathcal{X}}, \mathbb{Q}_{\ell}(1)\right)^{G_{k}} .
$$

L'article de Rosen et Silverman [14] décrit de nombreux exemples de surfaces (elliptiques) qui vérifient la conjecture 1.1. Nous rassemblons dans la remarque suivante la plupart des résultats connus ( $c f$. [13], [14]) en les ordonnant selon la classification des surfaces, notamment selon leur dimension de Kodaira notée $\kappa(\mathcal{X})(c f .[2]$ et [3]). 
Remarque 5.4. - Soit $\mathcal{X}$ une surface lisse et projective définie sur un corps de nombres $k$. La conjecture de Tate I est vérifiée dans les cas suivants :

1) La surface est rationnelle ou réglée $(\kappa(\mathcal{X})=-\infty)$.

2) La surface $\mathcal{X}$ est une surface K3, une surface d'Enriques, une surface abélienne ou une surface bi-elliptique $(\kappa(\mathcal{X})=0)$.

3) La surface est le produit d'une courbe de genre 1 par une courbe de genre $\geq 2(\kappa(\mathcal{X})=1)$.

4) La surface est un produit de courbes de genre $\geq 2$, une surface modulaire de Hilbert ou une surface de Fermat $(\kappa(\mathcal{X})=2)$.

La conjecture de Tate II et donc la conjecture (1.1) sont également vérifiées dans tous les cas suivants (la conjecture de Nagao généralisée est a fortiori vérifiée pour toute fibration d'une de ces surfaces au-dessus d'une courbe versions analytique et taubérienne) :

1) La surface est rationnelle ou réglée $(\kappa(\mathcal{X})=-\infty)$.

2) La surface est une surface K3 singulière ou de «type CM », une surface abélienne $\mathrm{CM}$ ou facteur d'une jacobienne de courbe modulaire $(\kappa(\mathcal{X})=0)$.

$3)$ Une surface qui est produit d'une courbe modulaire de genre 1 par une courbe modulaire de genre $\geq 2(\kappa(\mathcal{X})=1)$.

4) La surface est une surface modulaire de Hilbert ou une surface de Fermat $(\kappa(\mathcal{X})=2)$.

Dans le cas où $\kappa(\mathcal{X})=-\infty$ les classes de diviseurs engendrent $H_{\text {ét }}^{2}\left(\overline{\mathcal{X}}, \mathbb{Q}_{\ell}(1)\right)$. Donc l'application des classes est déjà un isomorphisme, en particulier les conjectures I et II de Tate sont satisfaites. Nous renvoyons à [13, p. 237] pour l'affirmation (et les références) que la conjecture de Tate I est connue pour les variétés abéliennes (Faltings), les surfaces K3 (Deligne), les surfaces modulaires de Hilbert (Murty-Ramakrishnan) et les surfaces de Fermat (Shioda).

Les résultats de Faltings entraînent également que la conjecture de Tate I est vraie pour un produit de courbes (voir par exemple [18, th. 5.2]). Ajoutons que, si $\mathcal{X}^{\prime} \rightarrow \mathcal{X}$ est un morphisme surjectif, alors la conjecture de Tate I pour $\mathcal{X}^{\prime}$ entraîne relativement aisément la conjecture de Tate I pour $\mathcal{X}$ (voir [18, th. 5.2]). Or les surfaces d'Enriques (resp. les surfaces bi-elliptiques) possèdent un revêtement par une surface K3 (resp. une surface abélienne). La conjecture de Tate I est donc vérifiée dans tous les exemples cités.

Nous renvoyons à [13, p. 242] pour l'affirmation (et les références) que la conjecture de Tate II est connue pour les surfaces abéliennes de type CM ou facteurs de jacobiennes de courbes modulaires (dû pour l'essentiel à Shimura), les surfaces modulaires de Hilbert (Murty-Ramakrishnan) et les surfaces de Fermat (Shioda). Le cas des surfaces K3 singulières est dû à Shioda, celui des surfaces K3 de «type $\mathrm{CM}$ » peut se déduire du cas des surfaces abéliennes. Le cas de produit de courbes modulaires peut être traité par la méthode de Rankin. Dans chaque cas on réussit à exprimer $L_{2}(\mathcal{X} / k, s)$ comme produit de 
fonctions $L$ associées à une forme modulaire ou à un caractère de Hecke et on sait donc que $L_{2}$ ne s'annule pas $\operatorname{sur} \operatorname{Re}(s)=2$.

Remarque 5.5. - 1) On appelle ici surface $\mathrm{K} 3$ de «type $\mathrm{CM} »$ celles pour lesquelles le motif est de type $\mathrm{CM}$, i.e., sa fonction $L$ est produit de fonctions $L$ associées à des Grössencharacter de Hecke. Pour une variété abélienne de type CM, Pohlman montre dans [12] que la conjecture de Tate est équivalente à la conjecture de Hodge pour ces variétés. De plus la conjecture de Hodge est vraie en codimension 1, d'après un résultat de Lefschetz. En particulier, elle est vraie pour les surfaces abéliennes de type CM. L'argument pour les surfaces K3 de type CM est similaire à [12].

2) Les surfaces de Fermat sont les hypersurfaces de $\mathbb{P}^{3}$ données par une équation $a_{0} x_{0}^{d}+a_{1} x_{1}^{d}+a_{2} x_{2}^{d}+a_{3} x_{3}^{d}=0$ (si $d \leq 3$ c'est une surface rationnelle, si $d=4$ c'est une surface $\mathrm{K} 3$ de «type $\mathrm{CM}$ » et si $d \geq 5$, c'est une surface de type général avec $\kappa(\mathcal{X})=2$ ).

Exemple 5.6. - Donnons un exemple concret en genre $g$ quelconque, essentiellement tiré de [15]. Soit $P(x)=x^{2 g+1}+p_{2 g} x^{2 g}+\cdots+p_{0}$ un polynôme séparable de $k[x]$, on définit alors la courbe $X$ sur $k(t)$ comme la courbe lisse projective dont un ouvert affine est donné par

$$
y^{2}=x^{2 g+1}+p_{2 g} x^{2 g}+\cdots+p_{0}+t^{2} .
$$

Si $f: \mathcal{X} \rightarrow \mathbb{P}^{1}$ est une fibration d'une surface projective lisse définie sur $k$ dont la fibre générique est $X$, on voit que $\mathcal{X}$ est $k$-rationnelle (en effet la surface est birationnelle à la surface d'équation $u v=P(x))$. Comme $\operatorname{Pic}^{0}(\mathcal{X})=0$, on a aussi $B=0$. Soit $O$ le point à l'infini de $X$ et $P_{i}=\left(e_{i}, t\right)$ (resp. $\left.P_{i}^{\prime}=\left(e_{i},-t\right)\right)$ les points de $X(\bar{k}(t))$ correspondant aux zéros de l'équation $P(x)=0$. On voit tout de suite que, si on plonge $X$ dans $J_{X}$ en prenant $O$ comme point base, on a les relations $P_{i}+P_{i}^{\prime}=0$ et $P_{1}+\cdots+P_{2 g+1}=0$. On sait par Shioda [15] que $\operatorname{rang}\left(J_{X}(\bar{k}(t))\right)=2 g$ et que les points $P_{1}, \ldots, P_{2 g}$ forment un système générateur. Supposons de plus $e_{i} \in k$, ou encore $P_{i} \in X(k(t))$ alors on a aussi $\operatorname{rang}\left(J_{X}(k(t))\right)=2 g$ et donc, dans l'exemple présent $A_{\mathfrak{p}}^{*}(\mathcal{X})=A_{\mathfrak{p}}(\mathcal{X})$ et on a

$$
\lim _{T \rightarrow \infty} \frac{1}{T}\left(\sum_{\substack{\mathfrak{p} \notin S \\ q_{\mathfrak{p}} \leq T}}-A_{\mathfrak{p}}(\mathcal{X}) \log \left(q_{\mathfrak{p}}\right)\right)=2 g .
$$

Si l'on ne suppose plus que $P$ a toutes ses racines dans $k$ on doit remplacer $2 g$ par le nombre de facteurs moins un dans la décomposition en facteurs irréductibles de $P$ dans $k[x]$.

Exemple 5.7. - Donnons un autre exemple avec cette fois une $\mathbb{Q}(t) / \mathbb{Q}$-trace non nulle, ce qui rend donc indispensable de considérer la trace moyenne réduite des Frobenius. Soit $X$ la courbe lisse projective définie sur $\mathbb{Q}(t)$ dont un ouvert 
affine est donné ainsi :

$$
U:=\left\{(x, y, z) \in \mathbb{A}^{3} \mid y^{2}-(x-a)(x-b)(x-c)=z^{2}-x(x-1)(x-t)=0\right\},
$$

où $a, b, c \in \mathbb{Q}^{*}-\{1\}$ sont distincts. Soit $V$ la surface affine lisse définie sur $\mathbb{Q}$ ainsi

$$
\begin{array}{r}
V:=\left\{(x, y, z, t) \in \mathbb{A}^{4} \mid y^{2}-(x-a)(x-b)(x-c)=z^{2}-x(x-1)(x-t)=0\right. \\
t \neq 0\}
\end{array}
$$

et munie de la fibration $f(x, y, z, t)=t$ de $V$ vers $\mathbb{A}^{1} \backslash\{0\}$. On peut construire une surface lisse projective $\mathcal{X} / \mathbb{Q}$ munie d'une fibration notée encore $f$ de $\mathcal{X}$ vers $\mathbb{P}^{1}$ telle que sa restriction à $V$ soit la fibration $f: V \rightarrow \mathbb{A}^{1} \backslash\{0\}$. La fibre générique de $f: \mathcal{X} \rightarrow \mathbb{P}^{1}$ est naturellement $X / \mathbb{Q}(t)$. Introduisons les courbes lisses projectives définies par l'équation d'un ouvert affine

$$
\begin{aligned}
E_{1} / \mathbb{Q} & : y^{2}=(x-a)(x-b)(x-c), \\
E_{2} / \mathbb{Q}(t) & : z^{2}=x(x-1)(x-t), \\
C_{1} / \mathbb{Q}(t) & : w^{2}=x(x-1)(x-t)(x-a)(x-b)(x-c) .
\end{aligned}
$$

Le genre de $E_{1}$ et $E_{2}$ est 1 , le genre de $C_{1}$ est 2 et le genre de $X$ est 4 . L'application $\pi: V \rightarrow E_{1}$ donnée par $\pi(x, y, z, t)=(x, y)$ a pour fibres des coniques. Ainsi la surface $\mathcal{X}$ est birationnelle à une surface réglée au-dessus de $E_{1}$ et la conjecture de Tate est donc vérifiée pour cette surface; de plus ceci montre aussi que $E_{1}$ est la variété d'Albanese de $\mathcal{X}$ et par conséquent également sa $\mathbb{Q}(t) / \mathbb{Q}$-trace car $E_{1}=E_{1}^{\vee}$. On peut montrer que $J_{X}$ est isogène à $E_{1} \times E_{2} \times J_{C_{1}}$. On a donc

$$
\operatorname{rang}\left(J_{X}(\mathbb{Q}(t)) / E_{1}(\mathbb{Q})\right)=\operatorname{rang}\left(E_{2}(\mathbb{Q}(t))\right)+\operatorname{rang}\left(J_{C_{1}}(\mathbb{Q}(t))\right) .
$$

Il est bien connu que $\operatorname{rang}\left(E_{2}(\mathbb{Q}(t))\right)=0$. En utilisant une 2-descente, on peut montrer que rang $\left(J_{C_{1}}(\mathbb{Q}(t))\right)=1$ (avec comme générateur d'un sous-groupe d'indice fini la différence des deux points à l'infini de $C_{1}$ ), pour un choix assez général de $a, b, c \in \mathbb{Q}^{*}$. Si l'on pose comme précédemment

$$
A_{\mathfrak{p}}^{*}(\mathcal{X}):=\frac{1}{q_{\mathfrak{p}}}\left(\sum_{c \in \mathbb{P}^{1}\left(\kappa_{\mathfrak{p}}\right)} a_{\mathfrak{p}}\left(\mathcal{X}_{c}\right)\right)-a_{\mathfrak{p}}\left(E_{1}\right)
$$

on obtient donc en appliquant le théorème 1.3 :

$$
\lim _{T \rightarrow \infty} \frac{1}{T}\left(\sum_{\substack{\mathfrak{p} \notin S \\ q_{\mathfrak{p}} \leq T}}-A_{\mathfrak{p}}^{*}(\mathcal{X}) \log \left(q_{\mathfrak{p}}\right)\right)=1 .
$$

Nous terminerons en notant deux corollaires du théorème 1.3.

Corollaire 5.8. - La conjecture de Tate pour les surfaces est équivalente à la conjecture analytique de Nagao (généralisée). 
Démonstration. - La conjecture de Tate entraîne la conjecture analytique de Nagao d'après le théorème 1.3. Inversement si $\mathcal{X} / k$ est une surface, elle est birationnelle à $\mathcal{X}^{\prime} / k$ possédant une fibration en courbes sur $\mathbb{P}^{1}$, donc la conjecture de Nagao analytique entraîne la conjecture de Tate pour $\mathcal{X}^{\prime} / k$ ( $c f$. proposition 4.1) et donc pour $\mathcal{X} / k$ d'après la proposition 5.3.

Corollaire 5.9. - La conjecture analytique de Nagao (généralisée) pour les fibrations au dessus de $\mathbb{P}^{1}$ entraîne la conjecture analytique de Nagao (généralisée) pour les fibrations au-dessus d'une courbe quelconque $C$.

Démonstration. - D'après l'argument précédent, la conjecture pour les fibrations sur $\mathbb{P}^{1}$ suffit à entraîner la conjecture de Tate, et cette dernière entraîne la conjecture analytique de Nagao.

Remerciements. - Les deux auteurs remercient l'accord Brésil-France 69.0014/01-5 pour son soutien financier et aussi l'IMPA pour sa chaleureuse ambiance scientifique; ils remercient également le rapporteur pour ses suggestions, notamment concernant la preuve du Lemme 3.2.

\section{BIBLIOGRAPHIE}

[1] Artin (M.) \& Winters (G.) - Degenerate fibers and reduction of curves, Topology, t. 10 (1971), pp. 373-383.

[2] BĂDEscu (L.) - Algebraic surfaces, Springer-Verlag, 2001, éd. roumaine, Suprafete Algebrice, 1981.

[3] Beauville (A.) - Surfaces algébriques complexes, Astérisque, vol. 54, Société Mathématique de France, 1978.

[4] Deligne (P.) - Conjectures de Weil I, Publ. Math. Inst. Hautes Études Sci., t. 43 (1974), pp. 273-307.

[5] — éd. - Cohomologie étale (SGA 4 1/2), Séminaire de géométrie algébrique du Bois Marie, Lect. Notes in Math., vol. 569, Springer, 1976.

[6] Harsthorne (R.) - Algebraic Geometry, Springer-Verlag, 1983.

[7] Lang (S.) - Algebraic Number Theory, Addison-Wesley, 1970.

[8] — Abelian Varieties, Springer-Verlag, 1983.

[9] Milne (J.S.) - Étale Cohomology, Princeton University Press, 1980.

[10] Nagao (K.) - Construction of high-rank elliptic curves, Kobe J. Math., t. 11 (1994), pp. 211-219.

$[11] \_, \mathbb{Q}(T)$-rank of elliptic curves and certain limits coming from local points, Manuscripta Math., t. 92 (1997), pp. 13-32.

[12] Pohlman (H.) - Algebraic cycles on abelian varieties of complex multiplication, Ann. of Math., t. 88 (1968), pp. 161-180.

TOME $133-2005-\mathrm{N}^{\mathrm{O}} 2$ 
[13] Ramakrishnan (D.) - Regulators, algebraic cycles and values of $L$ functions, in Algebraic K-Theory and Algebraic Number Theory, Contemp. Math., vol. 83, American Mathematical Society, 1989, pp. 183-307.

[14] Rosen (M.) \& Silverman (J.) - On the rank of an elliptic surface, Invent. Math., t. 133 (1998), pp. 43-67.

[15] Shioda (T.) - Mordell-Weil lattices for higher genus fibration over a curve, in New Trends in Algebraic Geometry, London Math. Soc. Lecture Notes, vol. 264, Cambridge University Press, 1999, pp. 359-373.

[16] TATe (J.) - Algebraic cycles and poles of zeta-functions, in Arithmetical Algebraic Geometry, Harper and Row, New York, 1965, pp. 93-110.

[17] _ On the conjectures of Birch and Swinnerton-Dyer and a geometric analog, in Dix exposés sur la cohomologie des schémas, North Holland, 1968, Sém. Bourbaki exposé n ${ }^{\circ}$ 306, pp. 189-214.

[18] - Conjectures on algebraic cycles in $\ell$-adic cohomology, in Motives (Seattle, WA, 1991), vol. I, Proc. Sympos. Pure Math., vol. 55, American Mathematical Society, 1994, pp. 71-83.

[19] Wazir (R.) - Arithmetic on elliptic threefolds, Compositio Math., t. 140 (2004), pp. 567-580. 